\title{
Chemically induced morphology change in cluster-based nanostructures
}

\author{
A. Lando ${ }^{1}$, N. Kébaïli ${ }^{1}$ a, Ph. Cahuzac ${ }^{1}$, C. Colliex ${ }^{2}$, M. Couillard ${ }^{2}$, A. Masson ${ }^{1}$, M. Schmidt ${ }^{1}$, and C. Bréchignac ${ }^{1}$ \\ 1 Laboratoire Aimé Cotton, CNRS, UPR 3321, Université Paris-Sud, Campus Orsay, 91405 Orsay Cedex, France \\ 2 Laboratoire de Physique des Solides, CNRS, UMR 8502, Université Paris-Sud, Campus Orsay, 91405 Orsay Cedex, France
}

Received 23 July 2006 / Received in final form 29 September 2006

Published online 24 May 2007 - (c) EDP Sciences, Società Italiana di Fisica, Springer-Verlag 2007

\begin{abstract}
Preformed clusters carrying surfactant are used as primary blocks for the building of nano structures. Self assembly of silver atom based clusters, soft landed on a HOPG surface, generates a large variety of new architectures depending on the nature and on the concentration of the impurities. Fractal shapes fragmented into multiple compact like islands, and chain like structures might be formed. A strong local enhancement of the silver atom mobility at the surface of islands is responsible for those morphology changes.
\end{abstract}

PACS. 61.46.-w Nanoscale materials - 81.07.-b Nanoscale materials and structures: fabrication and characterization

\section{Introduction}

Among recent progresses in the building of new architectures at the nano scale, is the use of preformed clusters as primary building blocks. Following the "bottom up" approach, it has been demonstrated that the self assembly of clusters deposited on a substrate, under well defined conditions, allows the building of some predetermined shapes of nanostructures [1-5]. One of the critical parameters driving the final morphology is the clusters size. Small sizes promote compact shapes whereas large ones are in favour of dendritic shapes, eventually fractal ones [3]. This evolution is governed by the ratio between two time constants: the interval time separating two successive impacts of impinging clusters on a growing island and the coalescence time for the impinging clusters to fuse into the island. Both constants exhibit different size evolution and the cross over, when it exists, determines a critical size for the shape change. This size dependence is an advantage when clusters are used instead of atoms for the synthesis of nanostructures. When atoms are used as building blocks, such a shape evolution could be achieved by changing either the substrate temperature, or the atom deposition flux. Another major benefit in the use of clusters lies in the possibility to include a small amount of impurities into that species [6]. This may promote both a drastic change in the diffusion constant of the clusters on the substrate and strongly activates the self diffusion at the surface of the island, leading to a fast relaxation process. The aim of this paper is to show how new classes of

\footnotetext{
a e-mail: mouari.kebaili@lac.u-psud.fr
}

nano morphologies might be constructed when deposited clusters carry impurities. This study has been performed using silver clusters containing either oxygen or a mixture of oxygen and molybdenum oxides as a surfactant.

\section{Experiment}

In our experiment, heterogeneous clusters are produced by reactive nucleation in a gas aggregation source [7]. Briefly, a pseudo Gaussian distribution of neutral clusters is deposited at low impact energy $(0.05 \mathrm{eV} /$ atom $)$ and at room temperature, on a cleaved HOPG graphite, in a high vacuum chamber $\left(10^{-9}\right.$ torr $)$. The cluster distribution is characterized using a "reflectron" time of flight mass spectrometer. The mean size is controlled by the source parameters and can be varied from one hundred up to one thousand of atoms. For most of the experiments related here a mean size of 500 atoms/cluster (mean diameter of $2.5 \mathrm{~nm}$ ) has been used. A crystal quartz micro balance measures the intensity of the incident neutral cluster beam and the deposition time determines the coverage. Typical fluxes are $10^{10}$ clusters $/ \mathrm{cm}^{2} \mathrm{~s}$. Coverage ranging from 1 up to 8 monolayers is achieved within few minutes.

Both the soft landing conditions and the weak substrate-clusters interaction preserve the whole structure of the impinging clusters. Diffusion on the substrate allows the creation of nucleation sites, either by the sticking on surface defects (heterogeneous nucleation) or by collisions between diffusing clusters (homogeneous nucleation). Subsequent growth leads to statistically distributed nano islands imaged using a Scanning Electron 
Microscope (SEM) after transferring the samples in air. We verified that this transfer does not affect the morphologies by transferring the samples under high vacuum conditions and imaging them with a non contact Atomic Force Microscope (AFM). The similarity between the images obtained from both microscopes shows that the transfer in air does not affect the morphologies at least during our observation time window.

In most cases the deposition procedure is a sequential one. By using a mask covering half of the graphite substrate, it is possible to deposit first pure silver clusters on the surface, and in a second step silver clusters containing impurities on one half of the surface. Then it is possible to use a reference deposition area for any kind of experiment, so that the effect of the impurities carried by the clusters is unambiguously put into evidence. This makes also possible, on the same HOPG substrate, the comparison between deposits corresponding to two degrees of impurities, two different coverages etc.

\section{Results}

\subsection{Morphologies at low degree of impurity}

In a first step pure silver clusters are deposited on the whole graphite surface at given average size, flux and coverage. Then the cluster flux is blocked and half of the sample is masked. Oxidized clusters are generated by mixing 0.2 to $1 \%$ of oxygen in the helium gas of the condensation source (reactive nucleation process [7]). One observes in the mass spectrum a minor modification in the ion peak species, only, showing the occurrence of oxidized clusters. In a second step, these heterogeneous species are deposited on half of the surface and for a comparable coverage to the pure clusters step. We have done numerous depositions for various impurity concentrations, fluxes and coverages. Figure 1 displays typical examples of SEM images of the island structures, and the corresponding high resolution mass spectra, obtained under these conditions. It shows that if islands obtained with pure silver atom cluster deposition exhibit fractal structures with branch width around $16 \pm 2 \mathrm{~nm}$, the deposition of clusters carrying impurities leads to fragmented islands, without any coarsening. The fragmentation rate increases with the impurity concentration. The relaxed fragments are regularly distributed along the fractal skeleton. They exhibit a compact shape. The fragment diameter distribution is very sharp, centered around $30 \mathrm{~nm}$. Moreover the auto correlation function shows that the nearest neighbour distance between fragments is well defined and equals $73 \pm 10 \mathrm{~nm}[6]$.

\subsection{Morphologies at high degree of impurity}

It is known that silver clusters can be only poorly oxidized in the presence of pure oxygen [8]. Under our experimental conditions two oxygen atoms can stick on a silver atom cluster only. In order to increase the number of oxygen atoms, we used molybdenum oxide $\mathrm{MoO}_{3}$ as
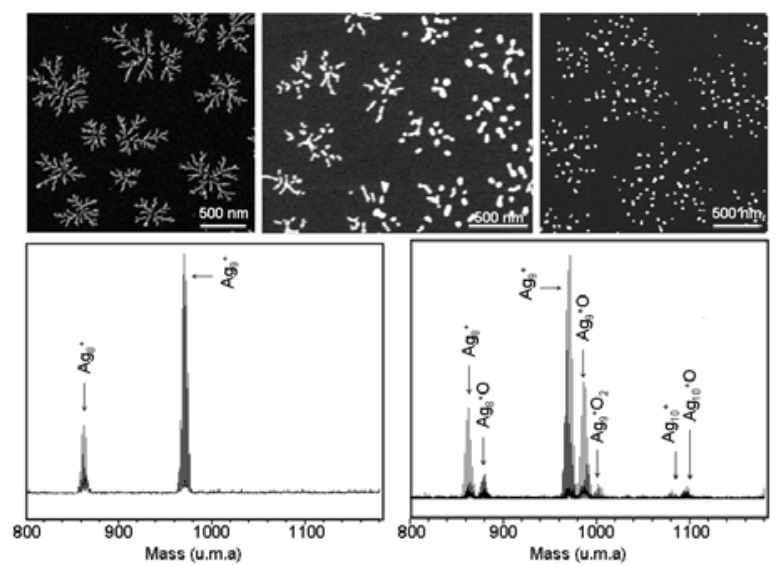

Fig. 1. Morphology change driven by the addition of oxygen at low concentration into the clusters, and the corresponding mass spectra. Left: fractal shape observed with pure silver atom clusters. Right: fragmented fractal when clusters carry oxygen. Medium: shadow effect due to the thickness of the mask showing a progressive oxygen concentration from left to right.

a mediator. Under these conditions, reactive nucleation process generates silver molybdates. Such compounds are currently used in metallurgy because of their remarkable technological applications: low friction, high temperature silver doped transition metal oxide coatings [9], Ag-based superionic glasses [10] etc. Using a molybdenum crucible in the gas aggregation source, we generate binary oxide clusters, easily discriminated in mass spectra, and which can be labelled $\mathrm{Ag}_{n-2 y}\left(\mathrm{Ag}_{2} \mathrm{O}\right)_{y}\left(\mathrm{MoO}_{3}\right)_{x}$ with $x=1-3$ and $y=x \pm 1$ [8]. At low impurity concentration, one observes island morphologies similar to the pure oxygen case, but above a critical quantity $\epsilon_{c}$, around $2-5 \%$ of oxygen, drastic changes both in the morphologies and island distributions are observed (Fig. 2). A large density of chainlike islands with pearled structure (linear or folded ones) is formed. Simultaneously, a strong modification in the mass spectra is observed. Ion peak signals of pure silver atom clusters, which predominate at low impurity concentration, decrease and mass spectra exhibit as predominant series the stoichiometric ion peaks $\mathrm{Ag}^{+}\left(\mathrm{Ag}_{2} \mathrm{MoO}_{4}\right)_{p}$ and $\mathrm{Ag}^{+}\left(\mathrm{Ag}_{2} \mathrm{MoO}_{7}\right)\left(\mathrm{Ag}_{2} \mathrm{MoO}_{4}\right)_{p}$. The detailed morphologies of these nanochains depend on the parameters of the cluster beam, but it can be noted that it exists a universal trends of their characteristic dimensions (Fig. 3). The lengths increase while the widths decrease, whatever the experimental parameters are. Increasing either the coverage or the degree of impurities leads to a decrease of the lengths and to an increase of the widths, whereas increasing the cluster flux increases the lengths and simultaneously decreases the widths. As a consequence there is a length to width correlation.

\section{Discussion}

The above discussed features are summarized in a $3 D$ diagram, shown in Figure 4. Let us focus first on the pure 

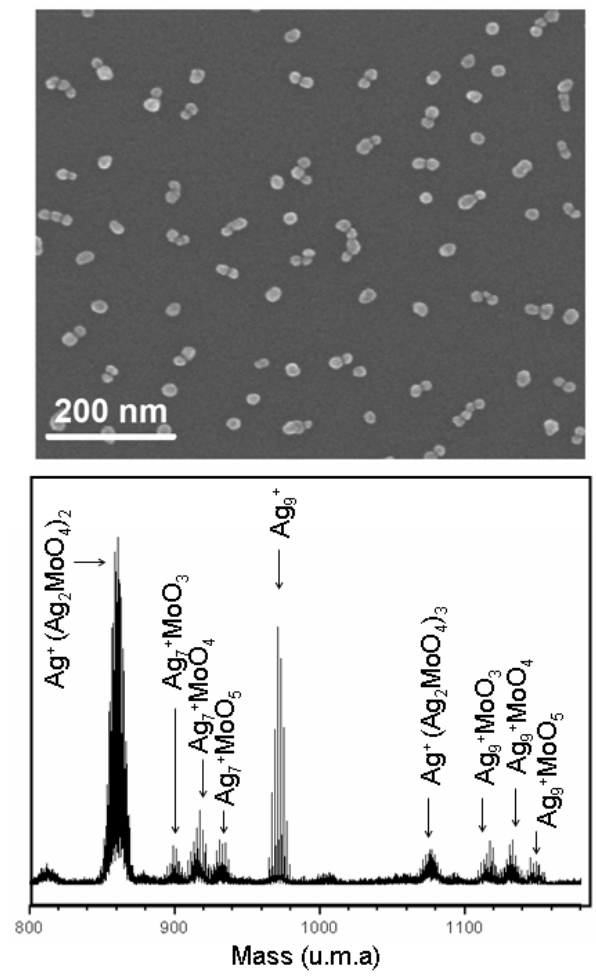

Fig. 2. Chain like nano structures observed with a high degree of impurity (binary oxides), and a typical corresponding mass spectrum.

silver atom cluster case. The island density, of the order of the natural defect density of an HOPG surface, indicates an heterogeneous nucleation process. This is expected if a large diffusion coefficient $D$ is considered. Taken into account the cluster flux, the capture area and the average atom number per cluster, a lower limit can be estimated: $D \geq 2 \times 10^{-7} \mathrm{~cm}^{2} \mathrm{~s}^{-1}$. The observed fractal structures tell us that for the cluster flux and the average size used here (typically: $0.2 \mathrm{MC} / \mathrm{min}, 500$ atoms/cluster), the coalescence time is long enough to enable predominating growth of non equilibrium fractal structures. This is in agreement with previous observations with antimony clusters [3] and with $C_{n}$ based films [11].

The fact, that when clusters carrying a small amount of impurity, a similar island density is observed, leads us to the conclusion that the surface cluster diffusion is not modified. On the contrary the relaxed shape into numerous of (compact) fragments, indicates that the self surface diffusion, i.e. the silver diffusion into the island, is strongly enhanced. This can be understood keeping in mind that in the bulk, the inclusion of oxygen strongly enhances the mobility of surrounding atoms, and that a migration towards the surface island is observed. A similar enhancement through the formation of $\mathrm{Ag}_{2} \mathrm{O}$ species has been evoked for pure silver nano particles deposited on $\mathrm{TiO}_{2}(110)$ or $\mathrm{Ag}(100)$ surfaces and exposed to a post deposition oxygen atmosphere [12-14]. More surprising is the difference between this chemically induced relaxation and a thermal induced relaxation of fractal islands [6].

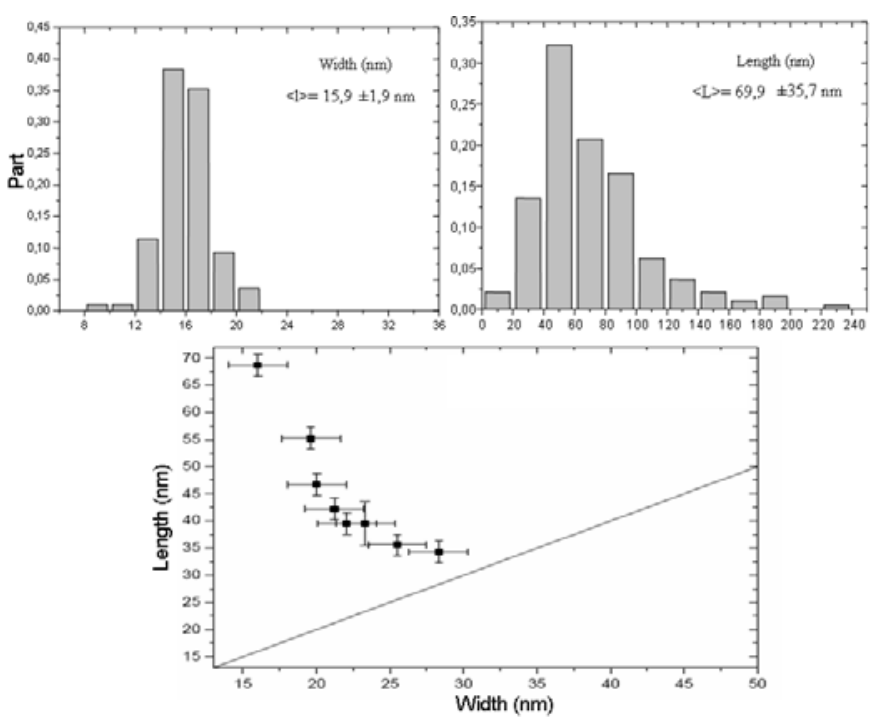

Fig. 3. Histograms for the width $l$ and the length $L$, of a given set of nanochains. Correlated evolution of $l$ and $L$ for samples obtained varying the cluster flux, the coverage and the impurity concentration.

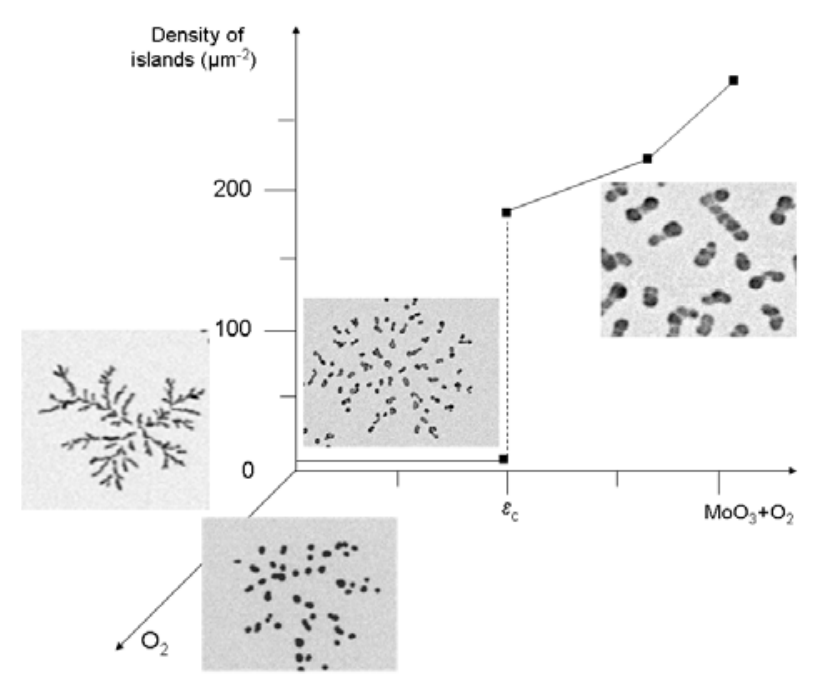

Fig. 4. Overview of the various morphologies built from silver atom based clusters and their dependence upon the nature and the concentration of impurities. The critical concentration $\epsilon_{c}$ corresponds to $2-5 \%$ of oxygen.

In this latter case, a coarsening of the fractal branches is observed first, which is not found here. Ultimately, the fragmentation generates a relatively small number of compact fragments with a very large disparity in size. As confirmed by Monte Carlo simulations (although done on 2D objects [15]), annealing promotes the enhancement of the whole surface atoms self diffusion of the island. Chemical impurities locally enhance this self diffusion, around oxidized centres, only. Moreover it is interesting to remember that, as shown on the histograms, the ratio between the nearest neighbour distance and the initial branch size is well defined and equals $4.6 \pm 1$ [6]. This value is in agreement with the value expected for the fragmentation 
of a non viscous column submitted to Rayleigh instabilities [16].

When a large amount of oxygen is carried by the clusters, through the presence of binary oxides, a large island density is observed. It increases proportionnaly to the coverage. It suggests an homogeneous growth process due to clusters-islands collisions. This implies that the diffusion of clusters on the graphite surface is lowered by several orders of magnitude as compared to the diffusion of pure silver clusters. An estimate from the saturated island density gives $D \leq 10^{-12} \mathrm{~cm}^{2} \mathrm{~s}^{-1}$. This is not surprising as the substrate-cluster interaction is increased by the presence of oxygen surface atoms and even more by an oxide sheath formation promoting dandling bonds. One notices that for a given deposition sample, the widths of the nano chains exhibit a sharp distribution whatever the lengths are, whereas the length distribution resembles to a Poissonian distribution according to a stochastic process (Fig. 3). The building via a strong anisotropic growth of such chain like islands is intriguing. At the present state of our investigations, no definite model emerges. Taking into account the characteristics of our gas aggregation source, one can rule out the growth of such ramified structures in the cluster beam itself, as it has been mentioned when a pulsed vaporization laser source is used or in case of $D C$ sputtering source $[17,18]$. However similarities exist with two processes leading to the building of nano wires : the Vapour-Liquid-Solid growth [19], known for making silicium whiskers for example, and Oxide Assisted Growth proposed to explain the growth of silicon nano wires or chains $[20,21]$. For this latter process, heterogeneous catalyst is not necessary. Oxidized nanoparticles nucleate and grow on a surface with phase separation between a metallic (inner) part and an oxidized (outer) one. This is accompanied with a recrystallization in order to minimize the free energy. Additional oxidized clusters land on the nascent island and growth continues along one direction driven by local instabilities. How is distributed in the final island the initial impurities carried by the cluster is still an open question. However, this image is supported here by the multi allotropic nature of the islands (Fig. 5). The pearled nature of the chains, could be explained by periodic change of the eutectoid mixture during the growth.

\section{Conclusion}

Large variety of morphologies can be built from a self assembly of clusters. Beyond the size effect which characterizes the use of preformed clusters as building blocks, the chemical composition can play a major role. The ultimate goal is obviously to use functionalized surfaces to elaborate well calibrated islands with a priori shapes on a perfectly regular structure and to look at the response of the structure to electromagnetic excitations.

\section{References}

1. L. Bardotti, P. Jensen, A. Hoareau, M. Treilleux, B. Cabaud, Phys. Rev. Lett. 74, 4694 (1995)

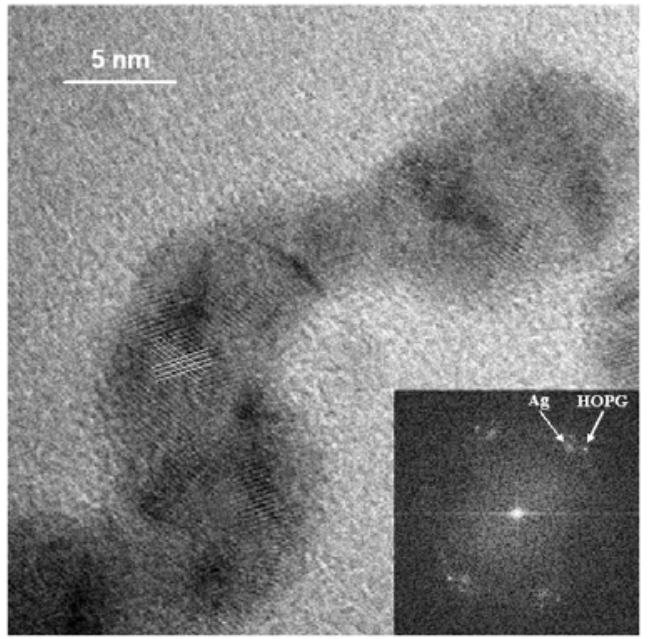

Fig. 5. HRTEM image of a nanochain. The interference fringes correspond to the (111) silver direction. Inset: corresponding Fourier transform pattern.

2. S.J. Carroll, K. Seeger, R.E. Palmer, Appl. Phys. Lett. 72, 305 (1998)

3. B. Yoon, V.M. Akulin, Ph. Cahuzac, F. Carlier, M. de Frutos, A. Masson, C. Mory, C. Colliex, C. Bréchignac, Surf. Sci. 443, 76 (1999)

4. B. Pawels, G. van Tendeloo, W. Bouven, L. Theil Khun, P. Lievens, H. Lei, M. Hou, Phys. Rev. B 62, 10383 (2000)

5. B. Stegemann, C. Ritter, B. Kaiser, K. Rademann, J. Phys. Chem. B 108, 14292 (2004)

6. A. Lando, N. Kébaïli, Ph. Cahuzac, A. Masson, C. Bréchignac, Phys. Rev. Lett. 97, 133402 (2006)

7. C. Bréchignac, Ph. Cahuzac, F. Carlier, M. de Frutos, J. Leygnier, J.Ph. Roux, J. Chem. Phys. 99, 6848 (1993)

8. C. Bréchignac, Ph. Cahuzac, N. Kébaïli, A. Lando, A. Masson, M. Schmidt, J. Chem. Phys. 121, 9617 (2004)

9. W. Gulbinski, T. Suszko, W. Sienicki, B. Warcholinski, Wear 254, 129 (2003)

10. St. Adams, J. Swenson, Phys. Rev. Lett. 84, 4144 (2000)

11. A. Böttcher, P. Weis, S.-S. Jester, D. Löffler, A. Bihlmeir, W. Klopper, M.M. Kappes, Phys. Chem. Chem. Phys. 7, $2816(2005)$

12. X. Lai, T.P. Stclair, D.W. Goodman, Faraday Discuss. 114, 279 (1999)

13. A.R. Layson, P.A. Thiel, Surf. Sci. 472, L151 (2001)

14. A.R. Layson, J.W. Evans, P.A. Thiel, Phys. Rev. B 65, 193409 (2002)

15. T. Irisawa, M. Uwaha, Y. Saito, Europhys. Lett. 30, 139 (1995)

16. J.W.S. Rayleigh, Proc. Lond. Math. Soc. 10, 4 (1879)

17. R. Alayan, L. Arnaud, M. Broyer, E. Cottencin, J. Lermé, J.L. Vialle, M. Pellarin, Phys. Rev. B 73, 125444 (2006)

18. S. Stappert, B. Rellinghaus, M. Acet, E.F. Wassermann, Eur. Phys. J. D 24, 351 (2003)

19. R.S. Wagner, W.C. Ellis, Appl. Phys. Lett. 4, 89 (1964)

20. N. Wang, Y.H. Tang, Y.F. Zang, C.S. Lee, S.T. Lee, Phys. Rev. B 58, R16024 (1998)

21. R.J. Barsotti Jr., J.E. Fischer, C.H. Lee, J. Mahmood, C.K.W. Adoo, P.C. Eklund, Mat. Res. Soc. Symp. Proc. 737, F.6.8.1 (2003) 